\title{
RADIATION FROM PLASMAS IN THE ST TOKAMAK
}

BY

\author{
N. BRETZ, D, DIMOCK, \\ A. GREENBERGER, E. HINNOV, \\ E. MESERVEY, W, STODIEK, \\ AND S, VON GOELLER

\section{PLASMA PHYSICS LABORATORY}

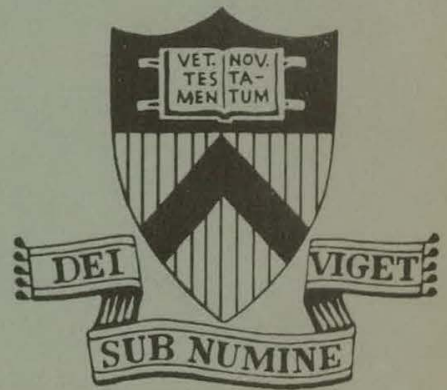

\section{PRINCETON UNIVERSITY PRINCETON, NEW JERSEY}

This work was supported by U. S. Atomic Energy Commission Contract AT (1)-1)-3073. Reproduction, translation, publication, use, and disposal, in whole or in part, by or for the United States Government is permitted. 


\section{DISCLAIMER}

This report was prepared as an account of work sponsored by an agency of the United States Government. Neither the United States Government nor any agency Thereof, nor any of their employees, makes any warranty, express or implied, or assumes any legal liability or responsibility for the accuracy, completeness, or usefulness of any information, apparatus, product, or process disclosed, or represents that its use would not infringe privately owned rights. Reference herein to any specific commercial product, process, or service by trade name, trademark, manufacturer, or otherwise does not necessarily constitute or imply its endorsement, recommendation, or favoring by the United States Government or any agency thereof. The views and opinions of authors expressed herein do not necessarily state or reflect those of the United States Government or any agency thereof. 


\section{DISCLAIMER}

Portions of this document may be illegible in electronic image products. Images are produced from the best available original document. 
IAEA-CN-33/A3-1

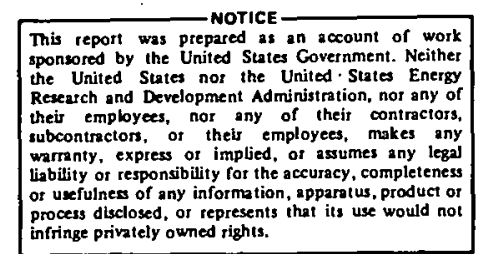

RADIATION FROM PLASMAS IN THE ST TOKAMAK *

N. Bretz, D. Dimock, A. Greenberger, E. Hinnov, E. Meservey, W. Stodiek, and S. von Goeler

Plasma Physics Laboratory, Princeton University, Princeton, New Jersey 08540, USA

Quanfitative vacuum ultraviolet (UV) measurements

$(\lambda \geq 100 \mathrm{~A})$ of various ST Tokamak discharges show that the atom density of metal impurities is about 0.18 to 1.58 of electron density and that the atom density of oxygen is about 18 to 108 . These concentrations yield an effective ionic charge, $\bar{z}$, consistent with the measured current, voltage, and profiles of electron temperature. The radial distribution of high-z material is obtained from $x$-ray measurements in the range $1 \leqslant \lambda \leqslant 4 A_{\text {; }}$ from these we conclude that there is no extreme concentration of high-z material in the center, but cannot exclude variations within a factor of 2. The $x$-ray spectrum is dominated by recombination; using bremsstrahlung and recombination calculations the value of $\bar{z}$ derived from $x$-ray intensity is comparable with the value from conductivity. An instrument is described for making measurements in the extreme UV region $(5<\lambda<20 \AA)$. In this range strong emission has been observed (radiated power about $10 \%$ of power input in the center). which we attribute to line emission from heavy metal ( $\mathrm{Fe}$ or Mo) ions which are produced only at electron temperature above about $1200 \mathrm{ev}$; the very narrow radial profile of observed intensity is consistent with measured temperature and density profiles. Rough estimates of the concentration of heavy metals required to account for the radiated power are consistent with vacuum $U V$ and $x$-ray data.

\section{INTRODUCTION}

The concentration and spatial distribution of high-z impurities promises to be of very great importance to the temperature, power balance, and stability of large tokamaks being built now or being planned for the future. In particular, if metallic impurities ( $\mathrm{Fe}, \mathrm{Mo}, \mathrm{W}$, for example). are present, the radiative power loss will be dominated by line radiation even at rather high electron temperature; only in very pure plasmas can 
one expect the limiting case of bremsstrahlung radiation balancing input power.

In this paper we attempt to correlate information about radiation intensities from ST Tokamak discharges as measured in $x$-ray, extreme ultraviolet (UV) and vacuum UV regions by a variety of instruments and methods. Aside from questions of mutual consistency of these measurements, the problem of particular interest is the evidence for or against preferential central concentration of heavy impurities, and, more generally, the importance of various processes in the energy balance near the center of the discharge.

The first section of the paper discusses analysis of measurements in the vacuum UV (100 $\AA \leqslant \leqslant \leqslant 1000 \AA)$, identification of ion species, and quantitative determinations of average impurity concentration; most of the power radiated from the ST appears in this general region. The second section of the paper discusses soft $x$-ray measurements $(1 \leq \lambda \leq 5 \AA)$ of bremsstrahlung and recombination radiation and their implications for the radial distribution of high-z ion species. The third section of the paper describes preliminary measurements with an instrument designed to explore the region. in the extreme UV $(5 \leq \lambda \leq 20 \AA)$ where we may expect, at higher temperature, emission of an important fraction of the power radiated from high-z ions, i.e., of total radiated power.

\section{VACUUM UV IDENTIFICATION OF IMPURITY ELEMENTS}

As in previous work. 1 the various ion species present are identified, and theix average concentrations are determined from absolute intensity measürements of specific ion resonance lines, by means of a grazing-incidence grating spectrometer. The lines appropriate for this purpose are those with $\Delta \mathrm{n}=0$, with $\mathrm{n}$ the principal quantum number of the ground configuration. These lines are generally the strongest, and occur in the wavelength range $(\lambda \geq 100 \AA)$ where the effective sensitivity of the grating spectrometer is highest. Furthermore, the excitation rate of such lines is practically independent. of temperature (for $T_{e} z 100 \mathrm{eV}$ ), and the rate coefficients are either known, or may be estimated with reasonable reliability.

Figure 1 shows a sample of such line intensities for various oxygen, molybdenum, and iron ions, together with the average electron density. The intensities are measured along the line of sight, in the central plane of the discharge, i.e., they represent

$$
J=\frac{1}{2 a} \int_{-a}^{a} n_{e}(r) n_{i}(r) s_{x}(r) d r=\frac{1}{2 a} \bar{n}_{e} \bar{s}_{z} \int n_{i}(r) d r
$$

where $s_{\text {is }}$ is the excitation rate coefficient, $n$ the electron density, and $n_{i}$ the population of the particular ion species. The last equation follows from the temperature-independence of $s_{x}$ and the relatively flat (uniform or parabolic) shape of $n_{e}(r)$. Often $n_{e}(x)$ [and $T(x)$ ] is measured independently by Thomson scattering, and this allows an estimate of the validity of this approximation. The last integral then gives the number of atoms in a particular state of ionization along the line of sight.

As is evident from Fig. 1, near the peak of a line only 2 or 3 states of a given element (perhaps 3 or 4 for heavier elements) are present at any one time, so that the total concentration of the element may be determined with fair accuracy. This method of ion density determination has been checked many times by adding known quantities of, e.g., neon or argon to the discharge. 
The changes in the electron density in Fig. I have no particular connection with the impurity concentration, but follow from changes of electron temperature and confinement time. The time of appearance and the duration of each of the lines are in general agreement with the separately known $n_{e}(r)$ and $T_{e}(r)$ time behavior, on the assumption that oxygen--and Fe and Mo às well--are present from the beginning of the discharge without substantial changes in total concentration.

The history subsequent to the initial intensity peaks of a particular element is somewhat more model-dependent. The sustained intensity plateau following the initial peaks must arise from more-or-less cylindrical shells of radiation sources located at radii where the electron temperature is comparable to (but somewhat lower than) the ionization potential. The constancy or near-constancy of these plateaus must indicate continued steady recycling of the element: neither an initial burst alone nor a rapidly increasing influx can be accommodated by the observations.

If one further assumes that the radial motion of an ion is slow (e.g.. $\sim$ plasma radius/mean particle containment time) then the state of ionization can be calculated as a function of radius from the known profiles $T_{e}(r)$ and $n_{e}(r)$. The observed plateau intensity then gives the rate of ionization, as well as the concentration of the ion in the shell. Such calculations show invarlably that a) tho oxygen concentration does not vary appreciably in time during the discharge and probably not in space; and b) the metallic impurities (for which the plateau measurement is usually very difficult) may show a slow increase in time, at least in some discharges.

From a set of such measurements, we can deduce the effective ionic charge, $\bar{z}=\Sigma n_{i} z_{i}^{2} / \Sigma n_{1} z_{i}$, and compare it with values determined from electrical resistance. The agreement is good, with the resistivity z-values generally 0-30z higher. The discrepancy may be due to unobserved impurities, such as tungsten (which cannot be measured at present because of the complicated and largely unknown electron configuration of its ions). Typical concentrations are: Oxygen, 3-10\%; molybdenum and iron, 0.1-1.0\%; plus traces of chromium and nickel, probably appropriate to the stainless steel wall composition. Generally there is a reciprocal relationship: with continued machine operation oxygen concentration falls and those of metals rise. The result is a gradual change in the radial profiles of $T_{e}(r)$ and other discharge parameters, but not necessarily of $\mathbf{Z}(\boldsymbol{r})$.

The highest states of ionization observed are close to, but slightly lower than those expected on the basis of coronal equilibrium (collisional ionization balanced by radiative and dielectronic recombination at measured $T_{\text {f }}$. Thus lithium-like and beryllium-like iron, and sodium-like and magnesium-like molybdenum have been observed. The existence of these states of ionization sets a lower limit to the ion confinement times, which turn out to be comparable to the average particle confinement times.

On the important question of relative concentration of heavy impurities toward the center (i.e., in the direction of electron density gradient) $)^{2}$ the UV measurements in the absence of radial scans can give no definite answer. However, plasmas with extreme concentration toward the center can be ruled out from energy balance considerations. Such plasmas would have high central resistivity, hence low current and power input density, and yet such large radiation losses that the steady-state temperature could not be maintained. On the basis of such considerations, a . neak-to-average variation of $\bar{z}(x)$ much larger than a factor 1.5 could not be maintained. 


\section{SOFT X-RAY DETERMINATION OF RADIAL DISTRIBUTION OF IMPURITIES}

In order to look more closely at the question of the radial distribution, and as a check on the UV measurements, we have made studies of the radial distribution of $x$-rays emitted by the plasma in the 3-10 kev range. This radiation is primarily recombination radiation of impurity ions plus a small amount of bremsstrahlung and $x$-ray line emission. This work has been discussed in detail elsewhere ${ }^{3}$; so we will give only a qualitative discussion here.

The recombination spectrum of a given ion consists of a sum lover final bound states) of bremsstrahlung-like spectra, each of which has a low-energy cutoff at the ionization energy of the particular final state. since the measurements were performed above the cutoff, each ion species appears simply to be radiating an enhanced bremsstrahlung spectrum. The strength of this radiation depends greatly on the elements present, on their states of ionization, and on the plasma temperature. For light impurities (in our case mainly oxygen) relatively accurate predictions can be made, because these elements become completely stripped in the central region of the discharge. For high $z$ impurities (mainly iron and molybdenum and/or tungsten) the ionization states must be calculated or measured. Both have been attempted for iron.

The measurement of ionization state consists of an investigation of the $\mathrm{k}_{\text {-line shift of iron. }}{ }^{4}$ The shift results from a reduction in shielding of the nuclear charge as electrons are removed from the L-shell in the process of ionization. Figure $2(a)$ shows a spectrum in the region 6.4 to $6.7 \mathrm{keV}$ obtained in a discharge with $1200 \mathrm{eV}$ central electron temperature. The instrument used was a commercially available Bragg spectrometer with a Lif crystal looking tangentially to the magnetic axis of the plasma. Its resolution can be deduced from the neutral iron $\mathrm{K}_{\alpha}-\mathrm{line--also}$ plotted in Fig. $2(a)$-which was produced by fluorescence from an $x$-ray tube. The indicated classification of the "satellites" follows that of Lie and Elton? The figure shows that for ST conditions ionization takes place in the L-shell, which contains 2 to 5 electrons at $\mathrm{T}_{\mathrm{e}}=1200 \mathrm{eV}$.

These measurements are compared with calculations balancing ionization and recombination. The computations of Jordan ${ }^{5}$ indicate that dielectronic recombination is the dominant recombination process with $\alpha_{\text {diel }}=10^{-11}$ to $3 \cdot 10^{-11} \mathrm{~cm}^{3} / \mathrm{sec}$ in the range of interest. These calculations, however, refer to the solar corona; at the higher densities of the tokamak, dielectronic recombination may be somewhat reduced. On the other hand, in the tokamak, the lifetime of the ions is important. Typical values of $1 / n \tau$, where $\tau$ is the particle confinement time, are in the range $10^{-11}$ to $18^{-12}$. Probably somewhat larger values should be used in a comparison with the recombination rate, because an ion recombines in the outer cooler plasma regions rather than at the wall. In Fig. $2(\mathrm{~b})$ is plotted the relative population of various ionization states as calculated by Jordan. 5 These calculations probably overemphasize the population of helium and lithiumlike states, which have smaller dielectric resombination and are probably determined more by particle loss. The relative intensities of the $\mathrm{K}_{\alpha}$ satellites can be determined from the concentrations, if the excitation ${ }^{a}$ functions are known. Assuming these all to be equal gives rough agreement with the observed spectrum.

Knowing the charge state of the ions, we can calculate the emitted recombination radiation and can make a comparison between the amounts of impurities deduced from the vacuum UV measurements and the $x$-rays. The measurements agree within a factor of 2 ; the $x$-rays tending towards somewhat higher concentrations than the vacuum UV. 
Representative x-ray data are shown in Fig. 3. Figure 3(a). shows Thomson scattering measurements of electron temperature and density profiles taken at a time $(70 \mathrm{msec})$ during the steady-state part of the discharge. In Fig. $3(b)$ the crosses $(x)$ show relative $x$-ray intensity (at $\lambda=3.1 \AA$ ) observed along chords at three different radii; the circles (o) are the corresponding relative radiation intensities calculated from the temperature and density profiles, assuming a uniform distribution of impurities. The normalizing factor for absolute intensity at the center is 50 times the $\mathrm{Z}=1$ bremsstrahlung intensity. Interpreted as recombination radiation, these data give $\overline{\mathrm{z}} \cong 8$, which agrees within the estimated accuracy with the conductivity result $(\overline{\mathrm{Z}}=7)$ and the vacuum ov data; therefore we conclude that the concentration of impurity is uniform to within a factor of 2 .

The $x$-ray measurements provide another kind of evidence about the impurity distribution. Phase and amplitude measurements of fluctuations in $x$-ray intensity ${ }^{6}$ clearly show the presence of $m=1, n=1$ oscillations in the central part of the plasma, extending out to a radius of $3 \pm 1 / 2 \mathrm{~cm}$. The theory of the $m=1, n=1$ MHD kink mode- shows that the radial extent of these oscillations will be bounded by the $q=1$. surface. Assuming the loop voltage to be independent of radius and using spitzer conductivity, we find the value of $\bar{z}$ within the central $3-\mathrm{cm}$ radius to be equal to $\bar{z}$ for the whole aperture within the experimental error of $\pm 50 \%$.

Our $x$-ray intensity profiles are similar to those in the T-4 Tokamak? which are attributed to impurity peaking in the center. This explanation does not apply in the ST plasmas. The difference is that the T-4 temperature profile (determined from $x$-ray measurements) is much broader than the ST profile (from Thomson scattering).

\section{EXTREME UV RADIATION INTENSITY AND RADIAL PROFILE}

Some data have been taken with a new diagnostic instrument designed to complement the $x$-ray and vacuum $U V$ data with measurements in the extreme UV range (5-20 ̊). This instrument consists of a windowless version of a commercially available self-scanned array ${ }^{8}$ of 128 silicon diodes, each measuring $\sim 0.005 \times 0.040 \mathrm{~cm}$. This array is placed behind a slit designed . for good space resolution; a set of thin metal filters arranged on a wheel gives some crude spectral resolution. The output of the array consists of a repeated series of 128 pulses each of which represents the charging current needed to replace the charge lost from a particular diode by exposure to radiation since the last time it was charged. Each pulse is therefore proportional to the integrated exposure of a diode for a period equal to the time required for a complete readout cycle. A charge sample and hold circuit ${ }^{9}$ provides a readable output for oscilloscope display. In our setup the external connections limited the operating frequency so that a complete cycle time was just under $1 \mathrm{msec}$.

The array was not expected to have much sensitivity beyond about $20 \AA$, since at longer wavelengths the radiation would be absorbed by the dead layer of the diodes and by the $1 \mu \mathrm{SiO}_{2}$ layer that is needed to prevent surface leakage. In addition, the array had a two-micron layer of $\mathrm{SiO}_{2}$ for scratch protection. Transmission curves for $\mathrm{SiO}_{2}$ and for several filters are shown in Fig. 4. These data are calculated from the tables of Henke, Elgin, et all.0 (The $\mathrm{SiO}_{2}$ actually contains some phosphorus, which is ignored in these calculations.) Because the $\mathrm{SiO}_{2}$ is in intimate contact with the diodes, the transmission curve represents a lower limit to the. effective transmission, since in our geometry some photons resulting from scattering and $x$-ray fluorescence go on to produce conduction charges in 
the diodes. Also some of the energetic electrons produced in the sio, reach the diodes; and there may also be a weak sensitivity at much longer wavelengths, due to fluorescent conversion into the visible region of the spectrum.

An approximate absolute calibration of the diode sensitivity was made as follows. The sensitivity of the array was measured in the visible at $6200 \AA$, using a standard lamp and a narrow-band interference filter. With a photoefficiency of $80 \%$, this can be converted to a sensitivity in terms of volts per electron-hole pair per $\mathrm{cm}^{2}$ per sec. Assuming that extreme UV photons produce electron hole pairs with about the same efficiency as somewhat harder x-rays (i.e., $\sim 3.5 \mathrm{eV}$ per pair) one has an energy calibration of the array in the extreme uv region.

Typical results are shown in Fig. 3 with data taken after a fairly extensive opening of the ST machine. Vacuum UV measurements for this particular discharge are not available, but past measurements show that such a discharge has oxygen as its dominant impurity, $\sim 108$, with smaller amounts of iron, $\sim 0.2 \%$, and molybdenum, $\sim 0.38$. The electrical behavior of the discharge and the electron temperature and density profiles show the character expected for this type of discharge.

In Fig. $3(b)$ the solid line shows the spatial distribution of the emission plotted versus chord radius. The dashed line is calculated as follows: from the electron temperature and density profiles, the $z=1$ bremsstrahlung intensity has been integrated from $0-15 \AA$, integrated along chords, and plotted against chord radius. This has been normalized to the observed intensity at the center, requiring a normalization factor of 400 .

The region of emission is extremely narrow, corresponding approximately to the flattopped region in the temperature profile. As discussed above, the $x$-ray measurements $(1<\lambda<4 \AA)$ indicate that this strong peaking cannot be attributed to a concentration of high-z material in the center. The possibility that we are seeing visible light from a reflection or incandescent spot on the vacuum wall is excluded, because the entire signal was eliminated by inserting a $0.5 \mathrm{~mm}$ piece of polished calcium fluoride, which cuts out the extreme UV and transmits the visible over the entire region where the diodes have any sensitivity to visible light.

The emission appears to be stronger and narrower than expected from bremsstrahlung or recombination radiation. We assume therefore that we are seeing line emission. Interpreting the observed radiation as line emission, we can account for the strong intensity peaking by assuming that the particular ion or ions we are observing occur only at temperatures above about $1200 \mathrm{eV}$. A crude determination of the energy of the emitted photons can be obtained from the absorbing filters. The $0.0013 \mathrm{~cm} \mathrm{Be}$ filter attenuates the signal by a factor of 4 , and the $0.0043 \mathrm{~cm} \mathrm{Al}$ by a factor of 8 . From the Be transmission curve in Fig. 4 we may conclude that most of the radiation we are seeing is to the long-wavelength side of $12 \AA$. Of the radiation transmitted by the Be filter, about half must lie in the region of the $\mathrm{Al} \mathrm{K}_{\alpha}$ absorption band from 4 to $8 \AA$.

In Figs. $5(a)$ and (b) are plotted, respectively, the diameter (full width at half height) and relative peak intensity of the emission versus time. It can be seen that at $30 \mathrm{msec}$ the width is even less than that shown in Fig. $3(\mathrm{~b})$ and increases in time. The radiation first appears at about the time when the center of the plasma reaches $1200 \mathrm{eV}$. At first. the profile width is about that of the $1200 \mathrm{eV}$ contour; later it broadens with time, but not as much as the $1200 \mathrm{eV}$ contour; this might be 
expected if the center intensity increases disproportionately because the increased temperature in the center brings in more lines at shorter wavelength. The increase in intensity with time is expected if the effective diameter of the radiating source increases as the temperature increases.

The exact origin of this extreme uV radiation cannot be determined without better spectral resolution; it is known, however, that L-shell lines of iron and $M$-shell lines of molybdenum lie in this region. Some L-shell iron lines in the range 10-17 A have been observed in solar flares ${ }^{11}$ and identified with states of ionization that correspond approximately to those deduced from the crystal spectrometer data of Fig. 2. No comparable data are available for molybdenum.

A crude estimate of the power radiated in the 0-15 $\AA$ region and of the number density of the emitting species can be obtained as follows. Using the known solid angle of the slit, we find that the peak emission is approximately $0.45 \mathrm{~W} / \mathrm{cm}^{2}$, i.e., $0.075 \mathrm{~W} / \mathrm{cm}^{3}$, using $6 \mathrm{~cm}$ as the diameter of the emitting column. This is a lower limit, since we know that most of the energy is being radiated in a spectral region where the silica layer can be expected to give significant attenuation:' If we assume that the observed radiation is concentrated near $12 \AA$ and make the naive assumption that we should correct for the full attenuation of the $3 \mu$ of silica, we get 0.5 $\mathrm{w} / \mathrm{cm}^{3}$. As nuted above, the fact that the silica is in direct contact with the diodes can be expected to reduce the attenuation, and $0.25 \mathrm{~W} / \mathrm{cm}^{3}$ can be considered to be a reasonable estimate for the emission in the spectral region 0 to $15 \AA$.

Using this value of radiated power ( $0.25 \mathrm{~W} / \mathrm{cm}^{3}$ ) and assuming a highly allowed $\Delta \mathrm{n}=1$ transition (the $11.9 \AA$ line of Fe XXII, for example) we calculate the number density of the emitting species to be $22 \times 10^{11} \mathrm{~cm}^{-3}$ ( $~ I z$ of electron density). In view of the crudeness of the estimate, this is a reasonable agreement with the vacuum $U V$ and $x$-ray measurements.

Further development of the silicon diode technique, coupled with crystal spectrometer measurements, can be expected to be very useful in analyzing the radiation in this important extreme UV region.

* Work supported by USAEC Contract AT (11-1)-3073.

[i] DIMOCK, D. L., et al., Nuclear Fusion 13 (1973) 271 .

[2] SPITZER, L., Jr., Ap. J. 116 (1952) 299.

[3] VON GOELER, S., et al., Princeton Plasma Physics Laboratory MATT-1081 (1974).

[4] LIE, T. N. and ELTON, R. C., Phys. Rev. A $\frac{3}{19}$ (1971) 865.

[5] JORDAN, C., Mon Not. R. Astr. Soc. 148 (1970) 17.

[6] VON GOFLER, S., et al., Princeton plasma physics Laboratory MATT-1058 (1974).

[7] VERSHKOV, V. A. and MIRNOV, S. V., Kurchatov Inst. Atomic Energy Report IAE-2298 (Moscow, 1973); also PPPL MATT-TRANS-113 (1973).

[8] RL 128A/17, Reticon Corp., Mountain View, California.

[9] RS-2C/128A, Reticon Corp., Mountain View, California.

[10] HENKE, B. L., et al., "X-Ray Absorption in the 2-to-200 $\AA$ Region," Nerelco Reporter XIV (No. 3-4):112, July-Dec. 1967.

[11] NEUPERT, W. M., et al., Ap. J. (Letters) 149 (1967) L79. 


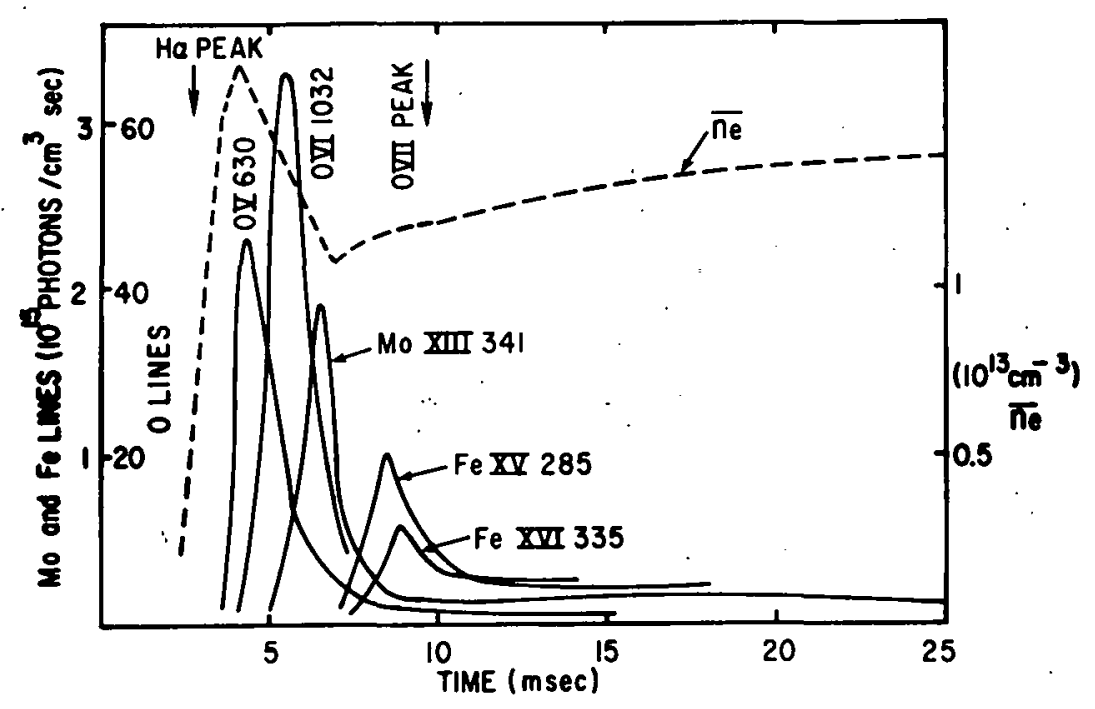

743644

Fig. 1. Time behavior of photon emission rate for vacuum UV lines of various ions. Dashed line is electron density from/microwave interferometer data, averaged over a diameter.

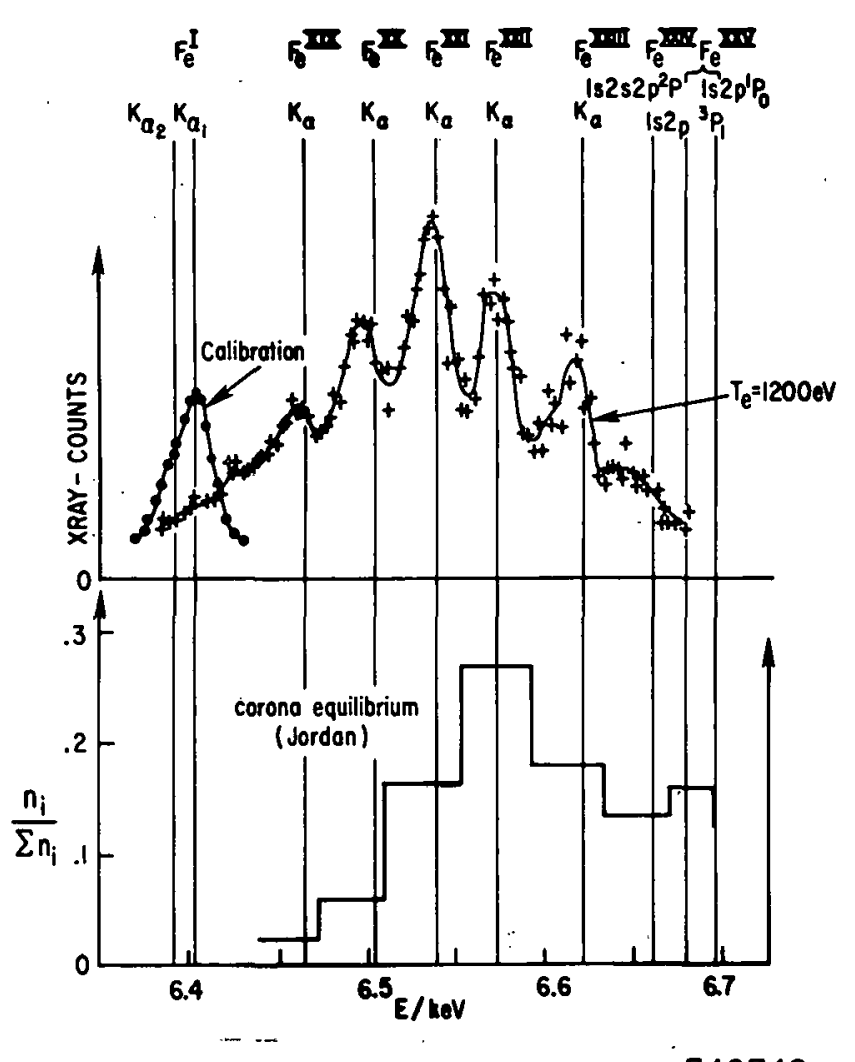

743749

Fig. 2. Spectrum of iron $\mathrm{K}_{\alpha}$ line structure. (a) Measured spectrum at central electron temperature $\mathrm{T}_{\mathrm{e}}=1200 \mathrm{eV}(+)$; neutral $K_{\alpha}$ line showing resolution of Bragg spectrometer $(\bullet)$; line identification from Ref. [4]. (b) Relative population of ionization states predicted by Jordan (Ref. [5]) for corona equilibrium at $\mathrm{T}_{\mathrm{e}}=1200 \mathrm{eV}$.
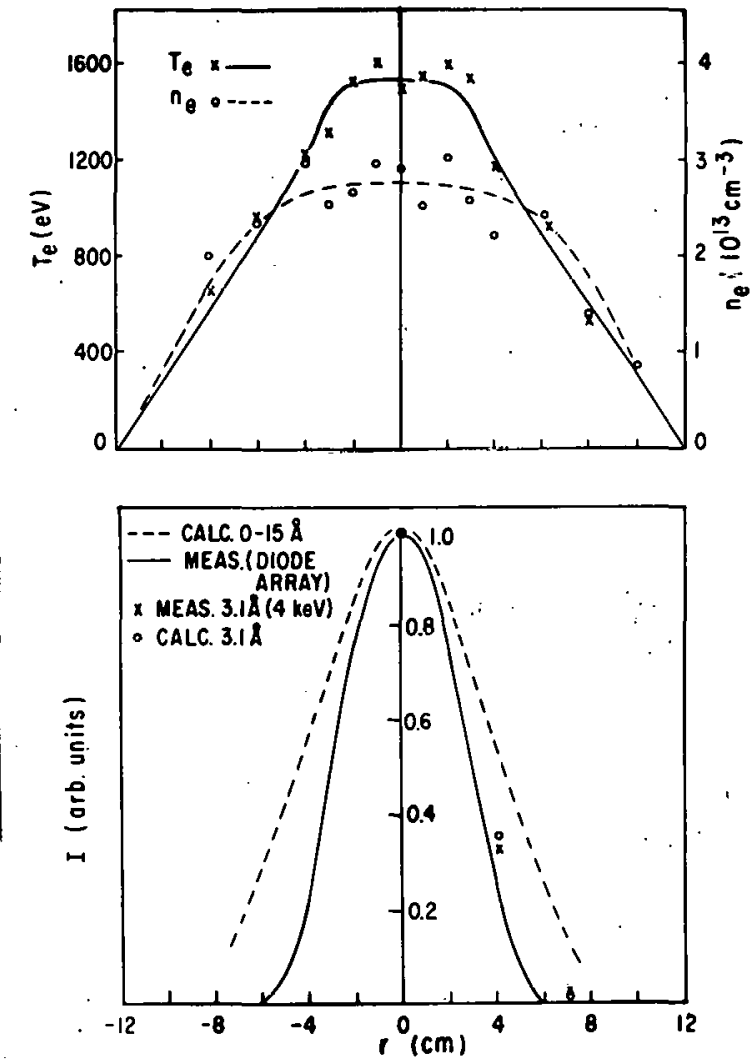

743125

Fig. 3. (a) Profiles of electron temperature and density vs radius. (b) Radiation intensities vs chord radius. Calculations are shapes expected for $\mathrm{z}=1$ bremstrahlung. Intensity normalizing factor is 50 for soft $x$-ray at $\lambda=3.1 \AA(0)$ and 400 for diode array (extreme UV) range $0-15 \&(---)$. 


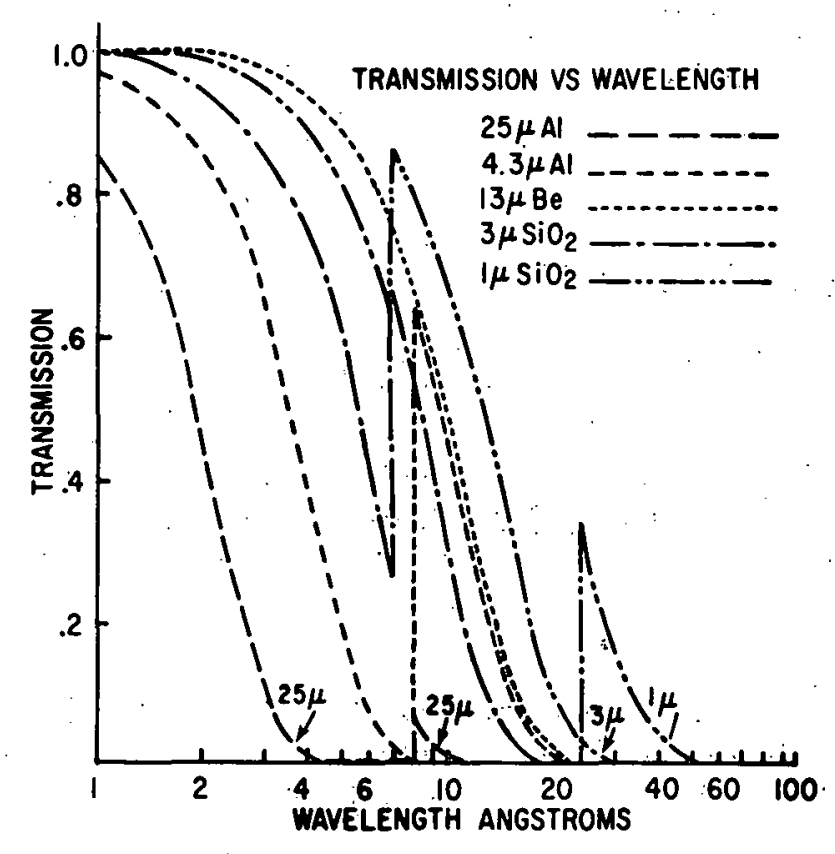

743643

Fig. 4. Transmission curves for $\mathrm{SiO}_{2}$ (detector surface coating) and for $\mathrm{Be}$ and $\mathrm{Al}$ filters.
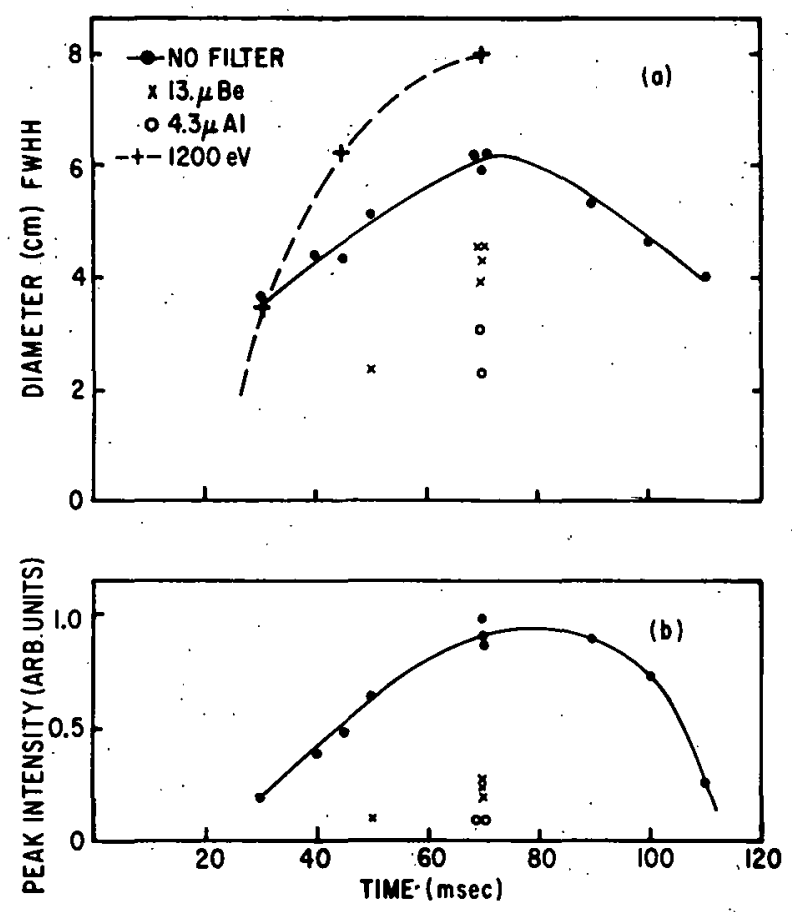

Fig. 5. (a) Time variation of full width at half height of extreme UV signal from silicon detector array. - no filter; $x 13 \mathrm{\mu} \mathrm{Be}$ filter; 0 4.3 H Al filter; + $1200 \mathrm{eV}$ contour diameter. (b) Relative peak intensity for same conditions as (a). 


\section{LEGAL NOTICE}

This report was prepared as an account of Government sponsored work. Neither the United States, nor the Commission, nor any person acting on behalf of the Commission:

A. Makes any warranty or representation, express or implied, with respect to the accuracy, completeness, or usefulness of the information contained in this report, or that the use of any information, apparatus, method, or process disclosed in this report may not infringe privately owned rights; or

B. Assumes any liabilities with respect to the use of, or for damages resulting from the use of any information, apparatus, method, or process disclosed in this report.

As used in the above, "person acting on behalf of the Commission" includes any employee or contractor of the Commission to the extent that such employee or contractor prepares, handles or distributes, or provides access to, any information pursuant to his employment or contract with the Commission. 
